\title{
Innovation Network Structure of Industrial Cluster of New Energy Vehicles in the Northeast China
}

\author{
Chen Wei ${ }^{1}$, Jing Rui ${ }^{1}$, Zhou Wen ${ }^{2}$, LIN Chaoran ${ }^{1}$ \\ ${ }^{1}$.School of Economics and Management, \\ Harbin Engineering University, Harbin 150001, China; \\ 2. College of Information System and Management, National University of Defense \\ Technology, Changsha 410073, China)
}

\begin{abstract}
The new energy vehicles industry is the strategic development direction of China's vehicles industry when there is an important need for this industry to realize the goal of energy conservation and environment protection, and of industry transformation and upgrading. It is an important approach that cooperatively innovating in the innovation network of industrial cluster to improve the industry's innovation level and core competitiveness. Based on the empirical investigation of the innovation network of industrial cluster of new energy vehicles in the northeast China, this paper uses complex network theory to study the network structure from the perspectives of whole network, community and ego network of Hub. Moreover, this paper further discusses the matching problem of Hubs' influence in the network. In addition, the relative suggestions about network governance on the innovation network of industrial cluster of new energy vehicles in the northeast China are proposed.
\end{abstract}

Keywords: industry of new energy vehicles; innovation network of industrial cluster; complex network; nodes influence

\section{Introduction}

China's industry of new energy vehicles began in the early 21 st century, the new energy automotive research project was included in China's "863" major scientific and technological issues during "Tenth Five-Year-Plan" in 2001. During the Eleventh FiveYear-Plan, according to China's "energy-saving and new energy vehicles" strategy, in order to enhance the core competitiveness of new energy automotive industry, the government highly concerned about the development of new energy vehicles, innovation and industrialization, and also continued to increase support for new energy vehicle industry, vigorously promote cooperation between enterprises and research institutions or universities. A complete new energy vehicle research \& development system and regional strategic layout had been built. Several new energy vehicle industry cluster emerged, in the drive under the strategy through cooperation and innovation to enhance the competitive advantage, various industrial clusters constitute the regional innovation network ${ }^{[1]}$.

With the expansion of innovation network size of new energy vehicle industry cluster, the cooperation and innovation activities are full of complexity between the networks, such as cooperation, research institutions, universities and other institutions, they have both independence and interdependence, stability and dynamic, overall cooperation and local competition, formal and informal relationships of cooperation and innovation, longterm and short-term differences in characteristics such as innovation goals ${ }^{[2]}$. Besides, the innovation network are still have some problems, such as cluster enterprise learning competition deterioration under the stimulation of internal and external environment, and problems on coordinate knowledge sharing and intellectual property protection ${ }^{[3]}$. 
Therefore, the industry of new energy vehicles innovation cluster network management is significant to improve the stability and continuity of the network, as well as promote collaborative innovation network body and enhance the core competitiveness of the industry. Among them, the cluster innovation network structure analysis is an effective network management to achieve the prerequisite and foundation.

Many Research have focused on the cluster innovation network structure, Cao Lili ${ }^{[4]}$ compared the various modes of network structure of industrial cluster. Rampersad et al. ${ }^{[5]}$ discussed the structural features in cluster form innovation network through empirical research on ICT, biotechnology and nanotechnology industry technology innovation network. Batterink ${ }^{[6]}$ and Guo Yuanyuan ${ }^{[7]}$ et al. study the role of science and technology intermediary and network location of cluster performance and impact based on the analysis of the innovation network structure. Gardet et al. ${ }^{[8]}$ analyzes the influence and role in promoting the development of network structures and cluster innovation networks main Hub through case studies. In the empirical basis on cluster innovation network structure, Lin Qiuyue et al. ${ }^{[9]}$ analyzed The cluster innovation network simulation, Zhang Yongan et al. ${ }^{[10]}$ studied the influences of the structure on the innovation cluster innovation network resource utilization. Capaldo et al. ${ }^{[11]}$ and Li Zhigang ${ }^{[12]}$ et al. discusses the relationship between cluster network structure and enterprises' innovation performance. Wang Song et al. ${ }^{[13]}$ analyze the relationship between the degree of cooperation, openness and growth performance in cluster innovation network cooperation in an uncertain environment. In addition, based on the study of cluster innovation network structure, many scholars had further studied the evolution of the cluster innovation network problems ${ }^{[14-16]}$.

The contents of the above document researched cluster innovation network characteristics and other in-depth aspect of research, laid an important foundation for the research on network governance etc. But there is a lack of research on the new energy vehicle industry cluster innovation network structure now, and research on cluster innovation network structure is mostly limited to the overall analysis, and lack of depth study of individual networks community structure and core body (called Hub). For the $R \& D$ activity of new energy vehicle industry have a higher demand for innovation ability and enterprise scale, the industrial cluster innovation network Hub and community structure have an important impact on the development and evolution of the network structure. Therefore, based on the empirical investigation of new energy vehicle industry cluster in northeast innovation network, this article use complex network theory, from the whole network, community structure, and Hub individual net three aspect to analysis of its structure, then the influence of Hub matching problems is discussed and propose governance policy recommendations on new energy vehicle industry cluster in northeast innovation network.

\section{Empirical Data and Research Methods}

Northeastern new energy vehicle industry cluster is one of the main cluster of new energy vehicle industry, these Hubs are including Changchun FAW Group, Hafei Vehicles Group and Huachen Group and other leading enterprises. These Hubs are the centers of the new energy vehicle, with the upstream and downstream enterprises in the industry supply chain, relevant research institutions and universities to cooperate in innovation, value chain reconfiguration, sharing and dissemination of knowledge and technology, the gradual evolution of industrial clusters innovation networks. This paper take Hub as the core enterprise, and based on the nomination of snowball sampling method, by carrying out questionnaires to these Hub's technical department heads and managers, designated the companies and research institutions which have a relationship of cooperation and innovation, such as universities or research institutions, and issued the same questionnaire to these enterprises and relevant department, to determine its 
cooperative relationship within the diameter of innovation. After weighed the depth of the search, the cost of the search and effectiveness of the data, the paper ultimately determine to use 6 level of the snowball sampling, and then sampling northeast new energy vehicle industry cluster innovation networks including 135 related companies, research institutions and universities, on this basis, obtain the relational data of the cooperation and innovation between these them through questionnaires. Put these subject into a set of network node, named it $\mathrm{N}$, put the relationship between them into a even edge set $\mathrm{E}$. Name the new energy vehicle industry cluster in northeast innovation network $G(N, E)$. Since the focus of this paper is to study the structure of the basic form of the relations of cooperation innovation networks, so here it is defined $G(N, E)$ as undirected weighted network.

To study the northeast new energy vehicle industry cluster innovation network structure in-depth, this paper analyzes the use of complex network theory, the overall network structure of the network, the largest network of associations and Hub's individuals subject network, including basic network structure indicators such as average computing degrees, network density, the average clustering coefficient, average path length, and community division and distribution network characteristics, the formula to calculate the network structure indicators please go to references. ${ }^{[17]}$ In addition, during the investigation were found that due to the new energy vehicle industry not only belong to the high-tech innovative industries, but also has a high demand for funds and technical skills. Although the industry has launched a wide range of cooperation and innovation activities, but the focus is highly dependent on a small number of Hub companies, Such as Changchun FAW Group. Therefore, Hub business influence analysis in new energy vehicle industry innovation cluster is an important content in research.

In the complex network theory, the characterization of the node influential commonly used indicator is the degree of the node, its value indicates the number of nearest neighbors of nodes in the network nodes. But recent studies show that the influence of the node can not be simply considered as the number of relationships, one should also consider the position of the nodes in the network. When the degree of the node is small, even if it has less number of relationships, but it is in the key position at the center of the network, which tend to have a higher influence, or vice versa. Therefore, the degree of matching between the degree and the network location reflects the size of influence of the nodes. To address this issue, Kitsak et al. ${ }^{[18]}$ proposed K-core decomposition method, by recursively remove all nodes in the network which is less than or equal to the value of $\mathrm{K}$, which corresponds to the definition K-core $\left(K_{s}\right)$. Figure 1 is an exploded schematic, wherein the K-core can divide the network into three different layers, whereby the nodes are classified according to the influence.

Although K-core decomposition consider the degree and location of the nodes of the network, but this method's results is rough, and no significant distinction with heterogeneous networks. To this end, Zeng et al. ${ }^{[19]}$ collect the information $K_{s}$ of nodes and information of nodes that removed, proposed improvements of K-core decomposition method: the Mixed Model (MDD)

$k(i)_{m}=k(i)_{r}+\lambda k(i)_{e}$

Among them, $k(i)_{m}$ represents the degree of mixing of $i$ after decomposition by $K_{s}$. $k(i)_{r}$ represents the remaining number of neighbor nodes. $k(i)_{e}$ represents the removed number of neighbors node after decomposition. $\lambda$ is the impact factor, with a range of $[0,1]$. 


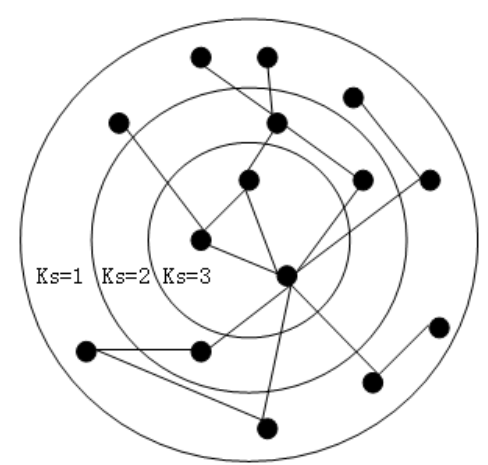

Figure 1. Decomposition Diagram of $K_{s}$

In order to further analyze the influence of Hub in the new energy vehicle industry cluster in northeast innovation network, this paper based on the matching condition of networks relationship and networks position, and by using improved $\mathrm{K}$ core decomposition method to compute the influence of main part of network. By matching the degree value with the main part of the network to determine the influence consistency degree of Hub.

\section{Empirical Analysis of Results}

The awareness and understanding of network structure is the premise and foundation, therefore, in order to deeply understand the structure of innovation network of northeast new energy vehicles industry cluster, on the basis of constructing the innovation network of new energy vehicle industry cluster in Northeast by empirical research data, this paper further studied the structural features of overall network, the largest community network and the Hub.

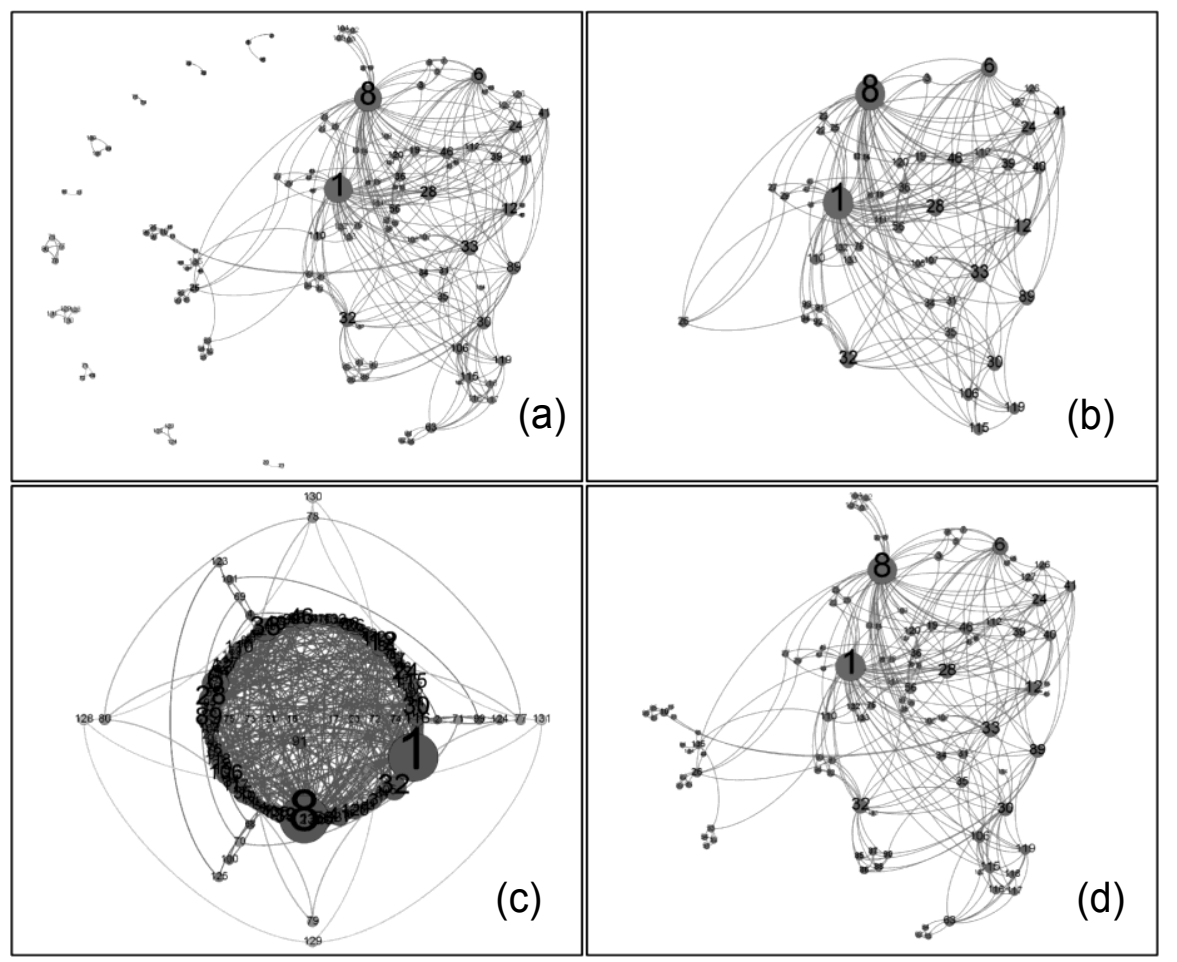

Figure 2. Northeastern New Energy Vehicle Industry Cluster Innovation Networks and Related Network Structure 
Figure 2 (a) use software Gephi to rendering northeast new energy vehicle industry cluster innovation network topology structure, figure 2 (b) is subject to the individual net of maximum value of the Hub (Changchun FAW Group). In addition, according to Newman's fast societies partitioning algorithm, we can divide northeast new energy vehicle industry cluster innovation network into 11 associations, figure 2 (c) is the results after dividing, in which each circle represents a community, figure $2(d)$ is the maximum communities network which was extracted from to figure 2(c). The basic structure of statistical index of northeast new energy vehicle industry cluster innovation network is as shown in Table 1, wherein the number within the brackets representing the percentage of the corresponding nodes accounted for the proportion of overall net.

Table 1. Northeast New Energy Vehicle Industry Cluster Innovation Networks of Basic Structural Statistical Indicators

\begin{tabular}{ccccccc}
\hline $\begin{array}{c}\text { Node } \\
\text { Type }\end{array}$ & Nodes & $\begin{array}{c}\text { Even } \\
\text { Edge }\end{array}$ & $\begin{array}{c}\text { Averag } \\
\text { Degree }\end{array}$ & Density & $\begin{array}{c}\text { Average coefficient } \\
\text { of agglomeration }\end{array}$ & $\begin{array}{c}\text { Average path } \\
\text { length }\end{array}$ \\
\hline Overall net & 135 & 388 & 5.75 & 0.04 & 0.38 & 2.84 \\
Maximum Associations Network & $107(79.26 \%)$ & $361(93.04 \%)$ & 6.75 & 0.06 & 0.39 & 2.81 \\
Individual network of Hub & $52(38.52 \%)$ & $234(60.31 \%)$ & 9.00 & 0.18 & 0.41 & 1.82 \\
\hline
\end{tabular}

In conjunction with Figure 2 and Table 1, the overall innovation network of new energy vehicle industry cluster in northeast are significantly heterogeneous, a small amount of Hubs has a lot of relations, and its network density is relatively sparse, lower average clustering coefficient, which indicates the network is still in the development stage, cooperation in innovation networks need to be further strengthened. In addition, Figure 3 is the distribution of innovation networks of northeast new energy vehicle industry cluster. Figure 3 shows the network is approximate obey power-law distribution $\mathrm{P}_{k} \propto e^{-1.92}$, it is a defender type that is similar to what Cai Ning et al. ${ }^{[20]}$ have proposed about three cluster network structure (market-based, defender type and hybrid networks). That means Hub are the center of the innovation network in new energy vehicle industry cluster in northeast, and the formation of clusters Hub leading many SMEs innovation model, research institutes and universities for the periphery, that new energy vehicle industry in northeast cluster innovation network Hub is mainly used in the body as the center, many small and medium enterprises, research institutions and universities to form the periphery of the cluster Hub leading innovation model that although the predevelopment of the cluster innovation network able to meet the new energy vehicle industry technology innovation for the enterprise high standards, but over-reliance on Hub easily lead to a high concentration of new energy vehicle industry technology innovation path dependence and Hub of knowledge of power, it is not conducive to sustained cluster innovation network's development ${ }^{[21]}$.

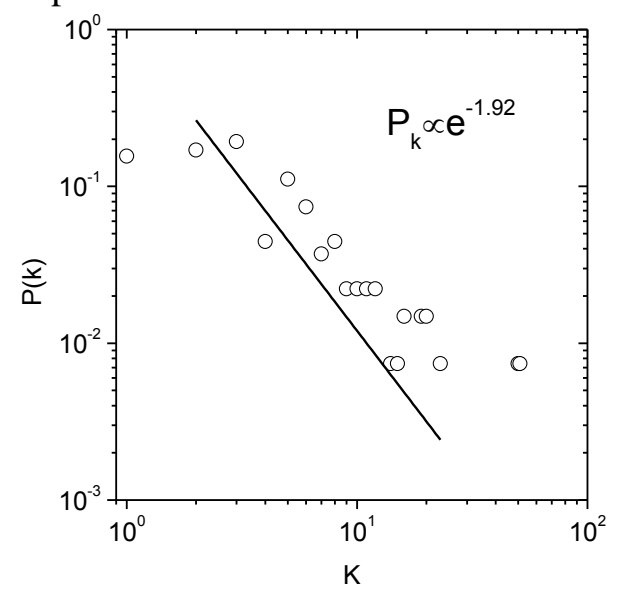




\section{Figure 3. Innovation Network Cluster Distribution of Northeast New Energy Vehicles Industry}

Although the new energy vehicle industry in northeast cluster innovation network can be divided into 11 associations, but from Figure 2(a) and 2(c), there is a huge community in the network can be seen, while the rest of the community small scale. Table 1 shows the largest community network node accounted for 79.26 percent of all the nodes of the network even accounting for 93.04 percent when considering edge even node, which indicates that most of the network subject in northeast can take good use of new energy vehicle industry cluster innovation network.

Because of the new energy vehicle industry cluster in northeast innovation network has a significant heterogeneity feature, Hub dominate the cluster innovation network, so we will focus on that influence. Figure 2(b) is the individual network of maximum degree enterprise (Changchun FAW Group), combined with Table 1, the number of neighbor nodes of the business accounted for 38.52 percent of all the nodes of the network, network connections edge accounting for $60.31 \%$ of all the number of edges connected to it, and compare with largest community network (figure 2(d)) shows that the maximum degree of an individual enterprise network covering more than half of the maximum basic community networks. This indicates that the maximum value of enterprises with a very wide range of cooperative relations innovation, and dominated most of the cluster innovation network cooperative innovation behaviors.

Due to the influence of the main network not only directly related to the number of network relationships, but related to the position in the network which occupied by the main network, the influence are determined by the matching of the degree and the network position. Therefore, this paper decomposition the northeast new energy vehicle industry cluster innovation networks by using the improved K-core decomposition method, and ultimately get 33 layers of K-core, wherein the highest level of K-core layer represents the network position of the node. Table 2 shows the matching of the influence of Hub, table 2 shows that Hubs are located in the same community, in addition, the ranking of the degree of three main networks (bolded in table 2) are not identical with the ranking of K-core, besides, others are identical. Especially these three abnormal nodes do not belong to the largest network or maximum K-core value layer. This shows that the influence of new energy vehicle industry cluster in northeast innovation networks Hub has remarkable match in terms of network relationships and network location.

Table 2. Innovation Network Hub Influential Matching of Northeast New Energy Vehicles Industry Cluster

\begin{tabular}{cccc}
\hline Nod No. & Network No. & Degree of Nod & $K$-core No. \\
\hline 1 & 1 & 51 & 33 \\
8 & 1 & 50 & 32 \\
6 & 1 & 23 & 31 \\
$\mathbf{3 2}$ & $\mathbf{1}$ & $\mathbf{2 0}$ & $\mathbf{2 9}$ \\
33 & 1 & 20 & 30 \\
12 & 1 & 19 & 28 \\
28 & 1 & 19 & 28 \\
30 & 1 & 16 & 27 \\
89 & 1 & 16 & 26 \\
24 & 1 & 15 & 25 \\
46 & 1 & 14 & 24 \\
41 & 1 & 12 & 23 \\
$\mathbf{1 0 6}$ & $\mathbf{1}$ & $\mathbf{1 2}$ & $\mathbf{2 1}$ \\
$\mathbf{1 1 5}$ & $\mathbf{1}$ & $\mathbf{1 2}$ & $\mathbf{2 1}$ \\
\hline
\end{tabular}




\begin{tabular}{cccc}
\hline 39 & 1 & 11 & 22 \\
40 & 1 & 11 & 22 \\
119 & 1 & 11 & 20 \\
35 & 1 & 10 & 19 \\
56 & 1 & 10 & 18 \\
63 & 1 & 10 & 18 \\
\hline
\end{tabular}

\section{Discussion}

In order to improve the level of competitiveness and innovation of new energy vehicle industry cluster innovation network, an important cooperative innovation model has been rapid development. In this paper, the new energy vehicle industry cluster in northeast innovation network become the empirical study subject, conducted in-depth study of its network structure. The results showed that $79.26 \%$ of the network in the main network have established relations of cooperation innovation in communication, and it accounted for $93.04 \%$ of all relations relationship, thus forming a huge communication societies (known as a giant-tuple), which not only means that most the main network can use cluster innovation network platform actively and conduct extensive cooperation and innovation, but also reflects the cluster innovation network acts a function of transform the cluster economy into a network connection. In order to improve their ability to innovate and to promote the sharing and dissemination of knowledge and technology, the need to build the innovation network and its effective governance for the network can be widely and effectively cooperate with other networks subject, and provides a good platform for innovation and cooperation.

Although empirical studies show that the new energy vehicle industry cluster in Northeast Innovation Network plays an important role in the whole, but through structural analysis we also found that the network density and average clustering coefficient is low, which means that the relationship between the main networks need to be further strengthened. In addition, studies show that Hubs such as Changchun FAW Group, Hafei Group and Huachen Group and other leading cluster innovation model. According to the theory of complex networks, for the stability of the network, which has significant heterogeneity structural in the network so that the stability of the network has dual characteristics ${ }^{[22]}$, on the one hand, it has a particular vulnerability when facing shocks, and the other hands it is robustness when facing non-specific aspects of risk. Considering the sharing of knowledge and technology in the network, although this network structure can be efficient when sharing knowledge and technology by Hubs ${ }^{[23]}$, but this is based on their knowledge and willingness, network connectivity and density as a precondition, so this kind of strong dependence is important in the early stage when knowledge and innovation capacity is needed. However, it will restrict the further development of network, especially leads to a strong main network dependence, technology development, path dependence, and highly centralized power of knowledge ${ }^{[24]}$, limited the diversity of the whole network.

\section{Countermeasures on Network Governance}

Network management of cluster innovation network is an important measure to achieve specific network functions through network restructuring and optimization. By researching on network structure of new energy vehicle industry cluster in northeast innovation, the results showed that the network structure is not optimal in both stability and ability to spread knowledge or technology, therefore, network management is important to enhancing its level of innovation and core competitiveness of new energy vehicle industry in the northeast innovation network cluster. In this paper, network management suggestions has been given to from three prospect, they are "cultivation 
medium-sized firms in cluster", "establishment of intermediary science and technology " and "establish and improve the flow of information science and technology intermediary mechanism".

(1) Cultivation medium-sized firms in cluster. Northeast new energy vehicle industry cluster innovation networks showed a significant heterogeneity currently, cooperative innovation activity is highly dependent on the Hubs, which is not conducive to enhancing the stability of the network structure of anti-risk impact and to improve continuity of knowledge and technology sharing and dissemination. Therefore, based on the network stability and knowledge and technology propagation angle, network management need to be considered from the network perimeter to cultivate the body or increase the number of middle-level main network, thereby reducing the difference of advantage of the connection between the Hub and the peripheral network, we need to cultivate mediumsized firms in cluster.

(2) Encourage the establishment of broad cooperation and innovation relationship. The results show that although the new energy vehicle industry in Northeast Cluster Innovation Network in most of the main network active use of new energy vehicle industry cluster in northeast innovation network platform to conduct extensive cooperation and innovation, but at present the network is still in the development stage, and its network density is sparse, its average cluster coefficient is low. Therefore, in order to promote the development of the cluster innovation network, to effectively play the innovation network to promote business cooperation. Cluster innovation platform's role is to encourage and support the establishment of a broad network of collaborative innovation body relationship, thereby increasing the density of cooperative innovation relationship.

(3) Establish the science and technology intermediary and improve information flow mechanisms. As technology and information flows mediation mechanism is full cooperation platform for innovation clusters innovation network acting basis and guarantee, in order to effectively encourage cluster innovation networks to establish broad cooperation and innovation subject relations and promoting enterprise network perimeter to quickly grow into medium-sized. Through the establishment of science and technology intermediary and improve the information flow mechanism to reduce the information asymmetry between the main networks and improve trust and collaboration of cooperative innovation is an important measure.

\section{References}

[1] Hu Dengfeng, Liu Jie, Cheng Nan. The Connotation, Evolution of the Innovation Network of the New Energy Automotive Industry and Its Orientation [J]. CHONGQING SOCIAL SCIENCES, 2012, (2): 9599.

[2] Wang Qin. ORCHESTRATING INNOVATION OF BUSINESS ECOSYSTEM OF EMERGING INDUSTRY: BASED ON ENERGY VEHICLES INDUSTRY [D]. Zhejiang University of Technology, 2013.

[3] Wang Zhanglin, Li Guofu, Zhang Fang. Under the perspective of improving intellectual property rights of China's new energy automotive industry policy [J]. Journal of Dalian Maritime University (Social Sciences Edition), 2014, (4): 18-20.

[4] Cao Lili. A Comparison Research on Networks Structure of Industrial Cluster [J]. China Industrial Economics, 2008, 29(10): 143-152.

[5] Rampersad G, Quester P, Troshani I. Managing innovation networks: Exploratory evidence from ICT, biotechnology and nanotechnology networks[J]. Industrial Marketing Management, 2010, 39(5): 793805.

[6] Batterink M H, Wubben E F, Klerkx L, et al. Orchestrating innovation networks: The case of innovation brokers in the agri-food sector[J]. Entrepreneurship and Regional Development, 2010, 22(1): 47-76.

[7] Guo Yuanyuan, Chi Zhiyong, Duan Na. Cost rise, inadequate foreign demand and the catch - up of manufacturing industries' sophistication: from the angle of native and foreign own and factor intensiveness heterogeneity [J]. Studies in Science of Science, 2014, 32(6): 841-851.

[8] Gardet E, Fraiha S. Coordination modes established by the Hub firm of an innovation network: The Case of an SME bearer[J]. Journal of Small Business Management, 2012, 50(2): 216-238. 
[9] Lin Qiuyue, Wang Wenping, Wang Jiaoli. Simulation Analysis of Innovation Network Structure of Industry Cluster Based on March 's Analytical Framework of Exploitation and Exploration [J]. Chinese Journal of Management, 2010, 7(7): 1015-1020.

[10] Zhang Yongan, Li Chenguang. Research on the Innovation Network Structural Impact on Innovation Resource Utilization [J]. Science And Management, 2010, 31(1): 81-89.

[11] Capaldo A. Network structure and innovation: The leveraging of a dual network as a distinctive relational capability[J]. Strategic management journal, 2007, 28(6): 585-608.

[12] Li Zhigang, Tang Shukun, Liang Xiaoyan, et al. An empirical study on the relationship between industry cluster's network structure and enterprises' innovation performance [J]. Studies in Science of Science, 2007, 25(4): 777-782.

[13] Wang Song, Sheng Ya. Cluster innovation network cooperation degree, network openness, and cluster incremental performance with the environmental uncertainty [J]. Science Research Management, 2013, 34(2): 52-61.

[14] Tian Gang, Zhang Yongan. Dynamical model and simulation for the evolution of industrial cluster innovation network [j]. Science Research Management, 2010, 31(1): 104-115.

[15] Ji Min, Hu Hanhui, Chen Jindan. Identification and rank confirmation of innovation platform [j]. Studies in Science of Science, 2011, 29(6): 861-867.

[16] Lv Guoqing, Zeng Gang, Ma Shuang, et al. Research on the compensation of technical human capital in China: issues, causes and solutions [j]. Studies in Science of Science, 2014, 32(9): 1423-1430.

[17] Wang Xiaofan, Li Xiang, Chen Guangrong. Introduction to Network Science [M]. Beijing: Higher Education Press, 2012.

[18] Kitsak M, Gallos L K, Havlin S, et al. Identification of influential spreaders in complex networks[J]. Nature Physics. 2010: 888-893.

[19] Zeng A, Zhang C J. Ranking spreaders by decomposing complex networks[J]. 2013: 1031-1035.

[20] Cai ning, huang chun. Risk and Structure Evolving of Industry Cluster: A Computer Simulation from Complex Network Perspective [J]. JOURNAL OF CHONGQING UNIVERSITY (Social Science Edition), 2012, (1): 5-11.

[21] LI zhidong, liu wen. Product Innovation:Complexity, Reliance and Networks [J]. Science \& Technology Progress and Policy, 2014, 31(17): 91-95.

[22] Albert R, Jeong H, Barabási A L. Error and attack tolerance of complex networks[J]. Nature, 2000, 406(6794): 378-382.

[23] Tsai K. Collaborative networks and product innovation performance: Toward a contingency perspective[J]. Research Policy, 2009, 38(5): 765-778.

[24] Shi Chengqi, Dang Xinghua. Knowledge-based innovation network evolution model of power [J]. Science \& Technology Progress and Policy, 2013, 30(17): 128-131.

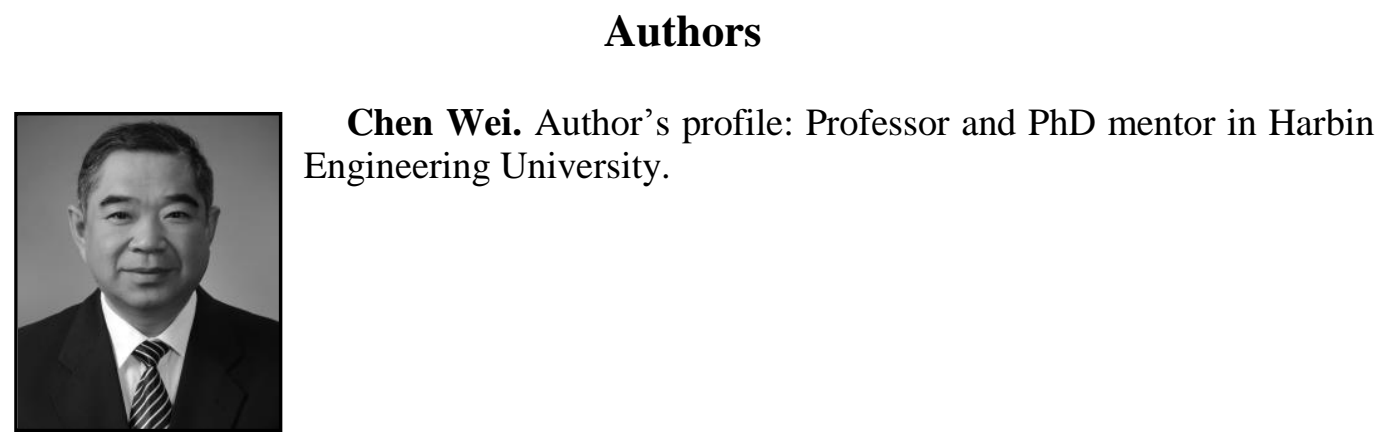


International Journal of Smart Home

Vol. 10, No. 6 (2016) 